\title{
Rémunération et congédiement des entraîneurs-chefs sur la performance d'équipes de sport professionnel : cas de la Ligue nationale de hockey (LNH)
}

\author{
Julien Bousquet ${ }^{a}$, Thierno Diallo ${ }^{b}$
}

RÉSUMÉ. L'identification des déterminants de la performance dans les sports professionnels permet aux gestionnaires de prendre des décisions opportunes pour atteindre de manière plus efficace les objectifs assignés. La présente recherche vise à mesurer la relation entre la performance des équipes de la LNH d'une part et, d'autre part, la rémunération des entraîneurs-chefs et leur congédiement. Les résultats issus du traitement des données secondaires nous permettent de dresser plusieurs constats. Par exemple, la performance des équipes de la LNH est corrélée négativement, mais non significativement avec le salaire des entraineurs-chefs. De plus, le congédiement de l'entrâ-neur-chef a quant à lui un impact significatif sur la performance de l'équipe à moyen terme. Ces résultats nous démontrent que les entraîneurs-chefs les mieux payés sont souvent engagés pour remonter les moins bonnes équipes.

\begin{abstract}
$\boldsymbol{A B S T R A C T}$. Identifying the determinants of performance in professional sports allows managers to make timely decisions to more effectively achieve assigned goals. The purpose of this research is to measure the relationship between the performance of NHL teams on the one hand and the compensation of head coaches and their dismissal on the other. The results from secondary data processing allow us to draw several conclusions. For example, the performance of NHL teams is negatively correlated, but not significantly with the salary of head coaches. In addition, the dismissal of the head coach has a significant impact on the team's performance in the medium term. These results show us that the highest paid head coaches are often hired to improve the performance of the worst teams.
\end{abstract}

\section{Introduction}

Les sports professionnels connaissent depuis deux décennies une popularité et une médiatisation importante. Les revenus engrangés par les équipes professionnelles sont astronomiques, et certains joueurs deviennent de véritables icônes. En ce qui a trait aux enjeux sportifs et financiers, les organisations sportives tentent par tous les moyens d'avoir une équipe gagnante et cela semble parfois passer par un entraîneur tout aussi gagnant (Mixon, Byrd et Wright, 2013). Plus récemment, l'attention s'est donc portée vers les entraineurs-chefs (Joaquin et Lago-Penas, 2010; Llorca et Teste, 2016), communément appelés « coachs » qui peuvent être tout aussi populaires et

\footnotetext{
a Professeur, Ph. D., Université du Québec à Chicoutimi

${ }^{\mathrm{b}}$ Professeur, Ph. D., Université du Québec à Chicoutimi
}

médiatiques que certains joueurs étoiles, et posséder des rémunérations tout aussi importantes. C'est le cas de José Mourinho, entraîneur de Manchester United en Premier League anglaise avec une rémunération annuelle, hors contrat publicitaire, de 28 millions d'euros (40 millions de dollars canadiens) ou de Mike Babcock, entraîneur-chef des Maple Leafs de Toronto, le mieux payé de la Ligue nationale de hockey, avec un salaire s'élevant à 6,25 millions de dollars canadiens.

Nous nous intéresserons donc dans le présent article aux entraîneurs-chefs des équipes de la Ligue nationale de hockey et leur possible impact sur le succès des clubs essentiellement parce que les données sont disponibles et parce que ce marché est le plus proche en termes de 
proximité et popularité au Québec. Cette recherche s'intéresse donc à deux hypothèses : (1) mesurer la relation entre la rémunération et la performance de l'équipe et; (2) mesurer la relation entre le changement de l'entraîneur et la performance de l'équipe.

L'article s'articule autour de cinq grandes sections. Les trois premières sont consacrées à la revue de la littérature, la problématique, la méthodologie utilisée. Finalement, les deux dernières présentent les résultats de l'analyse des données et les discussions et limites de la recherche.

\section{Revue de littérature : performance et entraîneurs-chefs}

Tout comme en management des organisations, beaucoup de chercheurs se sont intéressés à la performance des équipes sportives professionnelles depuis quatre décennies (Llorca et Teste, 2016; Lago-Ballesteros et Lago-Pena, 2010). Hormis l'aspect tactique de l'équipe (Slattery, Wallace, Bentley et Coutts, 2011) et les caractéristiques des joueurs (Bormann, SchulteCoerne, Diebig et Rowold, 2016), l'entraineur est devenu peu à peu une thématique étudiée fort intéressante dans la relation à la performance de l'équipe. Sous l'impulsion d'économistes (Llorca et Teste, 2016), les chercheurs se sont attardés à la dimension financière des équipes avec dans un premier temps la masse salariale, puis dans un second temps la thématique qui nous intéresse, à savoir la rémunération des entraîneurs d'équipes professionnelles.

L'entraîneur suscite donc une attention particulière de la part de la communauté universitaire depuis environ une vingtaine d'années puisque cet acteur jouit d'une popularité et une visibilité de plus en plus importante. De multiples recherches se ainsi sont penchées sur des thématiques s'intéressant aux opportunités professionnelles des entraîneurs (Knoppers, Meyer, Ewing et Forrest, 1991), aux facteurs psychologiques (Solomon, 2002), à la durée d'une carrière (Barros, Frick et Passos, 2009), au style de leadership (Vella, Oades et Crowe, 2013), et même plus récemment au genre (Darvin, Pegoraro et Berri, 2017). Et ce, sans oublier les deux hypothèses qui nous intéressent, à savoir la rémunération ou le salaire des entraîneurs (Grant, Leadly et Zygmont, 2013) et leur congédiement (Martinez et Caudill, 2013).

Concernant la rémunération, de manière surprenante, peu d'études empiriques se sont intéressées à la relation entre cette variable et la performance d'équipes professionnelles. La majorité d'entre elles se sont penchées sur les équipes universitaires (Colbert et Eckard, 2015; Grant, Leadly et Zygmont, 2013; Mixon, Byrd et Wright, 2013). Quelques-unes se sont aventurées dans l'univers du sport professionnel tel que la Ligue nationale de football aux États-Unis (Mondello, 2009), la Ligue nationale de hockey (Idson et Kahane, 2000) et finalement le championnat allemand de football, la Bundesliga (Frick et Simmons, 2008). Globalement, les résultats des recherches semblent démontrer une relation entre la rémunération d'un entraîneur et la performance des équipes professionnelles (Frick et Simmons, 2008; Mondello et Maxcy, 2009). Cependant, pour ce qui est du même type de recherche dans un contexte universitaire, les avis sont partagés. Certains chercheurs comme Colbert et Eckard (2015) déterminent une relation positive entre les deux variables, tandis que leurs collègues Mixon et Wright (2013) semblent affirmer le contraire malgré le même contexte d'étude (football universitaire américain), mais sur des périodes différentes.

Pour ce qui est du changement d'entraîneur, cette stratégie est censée relancer l'équipe (Balduck, Buelens et Philippaerts, 2010). Une fois encore, tous les résultats des principales recherches apparaissent mitigés, même si un consensus plutôt positif semble se dégager quant au lien potentiel entre le congédiement de l'entraîneur et la performance de l'équipe. Les recherches se sont toutes cantonnées aux sports professionnels, mais dans des disciplines différentes, du hockey en passant par le basketball. Selon Colbert et Eckard (2013), une tendance intéressante semble émerger de leur analyse. Le changement d'entraîneur pour un salaire plus élevé est automatiquement associé à l'amélioration de la performance à moyen terme, tandis que le remplacement pour un salaire inférieur ne semble pas significatif en matière de résultats. La seule étude démontrant clairement un lien fort entre les deux variables est celle menée par White, Persad et Gee (2007) au sujet de la Ligue nationale de hockey, tout comme l'échantillon auquel nous nous intéressons. La majorité des études (Balduck, Buelens et Philippaerts, 2010; Martinez et Caudill, 2013; Koning, 2003) présente des résultats très mitigés quant à la relation entre les deux variables. L'étude affichant la relation la moins significative entre ces deux variables, malgré le fait qu'elle ait été menée sur près de 80 années de données longitudinales (1920-2000), demeure à ce jour celle d'Andersen (2011). 
En guise de synthèse, les deux variables auxquelles nous nous intéressons, c'est-à-dire la rémunération et le congédiement d'un entraîneur, ont été passablement étudiées, mais ne semblent pas faire consensus quant à leur relation positive avec la performance. Bien que les résultats divergent et qu'aucune étude n'a pris en compte les deux variables conjointement, nous saisissons donc l'occasion pour explorer ces relations dans un contexte nord-américain de sport professionnel, celui de la Ligue nationale de hockey.

\section{Objectifs de la recherche}

L'objectif de cette recherche est de vérifier s'il existe une relation entre le niveau de performance des équipes de hockey et la rémunération des entraîneurschefs. De plus, nous nous intéresserons également à la relation entre le changement d'entraineur-chef et la performance de l'équipe. Cette recherche permettra alors de répondre à la question suivante : est-ce que le niveau de performance d'une équipe peut être influencé par le niveau de revenu ou par le remplacement de l'entraîneur. Pour réaliser la présente étude, deux hypothèses sont formulées (voir figure 1) :

H1 : Le niveau de salaire des entraîneurs-chefs a une relation significative sur la performance des équipes de hockey

\section{H2 : Le remplacement des entraîneurs-chefs a un impact sur la performance des équipes de hockey}

Nous nous attendons à avoir la présente relation entre les différentes variables.

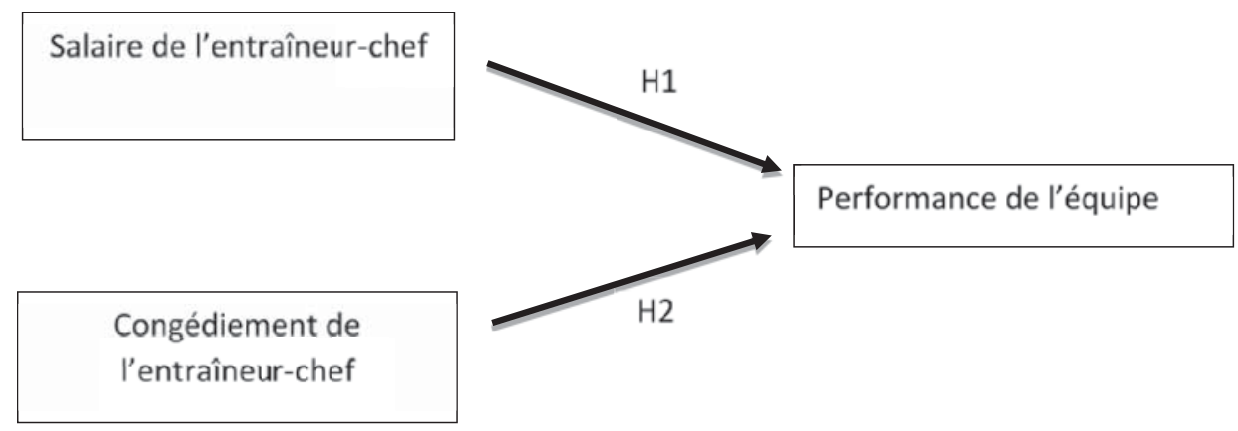

Figure 1- Hypothèses de recherche proposées

\section{Méthodologie}

Pour réaliser la présente recherche, trois séries de données ont été collectées sur cinq saisons de la Ligue nationale de hockey (LNH), de 2012 à 2107. Il s'agit des données relatives :

1- Au niveau de performance des équipes; le nombre de points de chaque équipe au cours de la saison nous permet de mesurer la performance. C'est une variable numérique de type échelle.

2- Aux salaires des entraîneurs-chefs; les salaires annuels bruts en dollars américains disponibles pour certaines saisons. C'est une variable numérique de type échelle.

3- Aux noms des entraîneurs-chefs des équipes au cours de la saison; l'entraîneur-chef initial (congédié ou non) prend la modalité 1 et son remplaçant au cours de la saison suivante prend la modalité 2 . Cette information est collectée pour documenter la variable relative au remplacement des entraîneurs et elle est une variable numérique de type nominale.

4- Au pays d'installation de l'équipe; la modalité 1 pour une équipe installée aux États-Unis et la modalité 2 pour une équipe installée au Canada. C'est une variable numérique de type nominale.

La constitution de cette base de données secondaires a été réalisée grâce au croisement des informations collectées sur les différents sites Internet de hockey. Les sites les plus utilisés pour collecter ces informations sont, entre autres, le site de la $\mathrm{LNH}$ (https://www.nhl.com/fr) et les sites indépendants de compilation d'informations sportives Cap Friendly (https://www.capfriendly.com) et Other League (http://www.otherleague.com). Toutes les données ont été croisées au minimum par deux sources différentes afin de s'assurer de leur exactitude. 


\section{Résultats des analyses de données}

\subsection{Salaire et performance de l'équipe}

Pour éliminer l'effet du pays d'installation de l'équipe sur la performance en raison des conditions économiques différentes - la fiscalité entre autres -, nous regardons s'il existe une différence significative entre la performance des équipes de la
LNH installées aux États-Unis et au Canada. Il est facile de constater dans le tableau 1 que les équipes installées au Canada ont en moyenne un niveau de performance $(87,15)$ supérieur à celui d'équipes installées aux États-Unis $(83,07)$. Cependant, le test T du tableau 2 ne montre pas de différence significative dans la performance (Sig. ,242) à $5 \%$ selon les lieux d'installation des équipes.

\begin{tabular}{ll|r|r|r|r}
\multicolumn{3}{c}{ Statistiques de groupe } \\
& Pays d'origine & $N$ & Moyenne & Ecart type & \multicolumn{1}{c}{$\begin{array}{c}\text { Moyenne } \\
\text { erreur } \\
\text { standard }\end{array}$} \\
\hline Performance équipe & États-Unis & 74 & 83,07 & 23,355 & 2,715 \\
\cline { 2 - 6 } & Canada & 68 & 87,15 & 17,225 & 2,089 \\
\hline
\end{tabular}

Tableau 1 - Statistiques descriptives performance - pays d'installation

\begin{tabular}{|c|c|c|c|c|c|c|c|c|c|c|}
\hline \multicolumn{11}{|c|}{ Test des échantillons indépendants } \\
\hline & & \multicolumn{2}{|c|}{$\begin{array}{c}\text { Test de Levene sur l'égalité } \\
\text { des variances }\end{array}$} & \multicolumn{7}{|c|}{ Test t pour égalité des moyennes } \\
\hline & & \multirow[b]{2}{*}{$\mathrm{F}$} & \multirow[b]{2}{*}{ Sig. } & \multirow[b]{2}{*}{$\mathrm{t}$} & \multirow[b]{2}{*}{ ddl } & \multirow[b]{2}{*}{ Sig. (bilatéral) } & \multirow{2}{*}{$\begin{array}{c}\text { Différence } \\
\text { moyenne }\end{array}$} & \multirow{2}{*}{$\begin{array}{l}\text { Différence } \\
\text { erreur } \\
\text { standard }\end{array}$} & \multicolumn{2}{|c|}{$\begin{array}{l}\text { Intervalle de confiance de la } \\
\text { différence à } 95 \%\end{array}$} \\
\hline & & & & & & & & & Inférieur & Supérieur \\
\hline \multirow[t]{2}{*}{ Performance équipe } & $\begin{array}{l}\text { Hypothèse de variances } \\
\text { égales }\end{array}$ & 15,761 &, 000 & $-1,176$ & 140 & .242 & $-4,079$ & 3,469 & $-10,937$ & 2,779 \\
\hline & $\begin{array}{l}\text { Hypothèse de variances } \\
\text { inégales }\end{array}$ & & & $-1,191$ & 133,888 & .236 & $-4,079$ & 3,425 & $-10,855$ & 2,696 \\
\hline
\end{tabular}

Tableau 2-Test $\mathrm{T}$ performance - pays d'installation

En ce qui concerne les statistiques descriptives, nous constatons que la moyenne des salaires des entraîneurs-chefs est de 2310393 \$. Nous observons une très grande dispersion des salaires des entraîneurs-chefs avec un écart-type de 1188495 \$ (tableau 3). Le tableau 4 de la corrélation entre salaire et performance de l'équipe ne montre cependant aucune corrélation significative à $5 \%$ entre ces deux variables (Sig. ,158). Pour les cinq saisons de la Ligue nationale de hockey (LNH), de 2012 à 2107, le salaire de l'entraîneur-chef ne semble pas avoir influencé la performance des équipes au cours des différentes saisons.

\begin{tabular}{l|c|c|c|c|c}
\multicolumn{5}{c}{ Statistiques descriptives } \\
& $N$ & Minimum & Maximum & Moyenne & Ecart type \\
\hline Salaire du coach & 89 & $\$ 800,000$ & $\$ 6,250,000$ & $\$ 2310393.26$ & 1188495.808 \\
\hline N valide (liste) & 89 & & & & \\
\hline
\end{tabular}

Tableau 3 - Statistiques descriptives 


\begin{tabular}{llrr} 
& \multicolumn{1}{c}{ Corrélations } & & \\
& & $\begin{array}{c}\text { Salaire du } \\
\text { coach }\end{array}$ & $\begin{array}{c}\text { Performance } \\
\text { équipe }\end{array}$ \\
\hline Salaire du coach & Corrélation de Pearson & 1 &,- 151 \\
\cline { 2 - 4 } & Sig. (bilatérale) & &, 158 \\
\cline { 2 - 4 } & $\mathrm{N}$ & 89 & 89 \\
\hline Performance équipe & Corrélation de Pearson &,- 151 & 1 \\
\cline { 2 - 4 } & Sig. (bilatérale) &, 158 & \\
\cline { 2 - 4 } & $\mathrm{N}$ & 89 & 89 \\
\hline
\end{tabular}

Tableau 4 - Test de corrélation salaire entraîneur - chef-performance

\subsection{Remplacement d'entraîneur-chef et performance de l'équipe}

Pour recruter les meilleurs entraîneurs dans le but de remonter les équipes dans le classement général, les équipes bonifient les salaires considérablement. En effet, le tableau 5 indique que les entraîneurs remplaçants sont en moyenne mieux payés (3 011111 \$) que les entraîneurs-chefs initiaux (1 888888 \$). Le tableau 6 ci-dessous montre une différence significative à 5 \% (Sig ,019) entre le salaire de l'entraîneur-chef initial et celui de son successeur.

\section{Statistiques de groupe}

\begin{tabular}{l|l|r|rr|r} 
& & & & & \multicolumn{1}{c}{$\begin{array}{c}\text { Moyenne } \\
\text { erreur } \\
\text { standard }\end{array}$} \\
\hline Salaire du coach & Changement de coach & $N$ & Moyenne & Ecart type & 150871,951 \\
\cline { 2 - 6 } & Entraineur initial & 9 & 1888888,89 & 452615,854 & 1503610,251 \\
\hline
\end{tabular}

Tableau 5 - Statistiques descriptives salaire entraîneur-chef - changement

\begin{tabular}{|c|c|c|c|c|c|c|c|c|c|c|}
\hline \multicolumn{11}{|c|}{ Test des échantillons indépendants } \\
\hline & & \multicolumn{2}{|c|}{$\begin{array}{c}\text { Test de Levene sur l'égalité } \\
\text { des variances }\end{array}$} & \multicolumn{7}{|c|}{ Test t pour égalité des moyennes } \\
\hline & & \multirow[b]{2}{*}{$\mathrm{F}$} & \multirow[b]{2}{*}{ Sig. } & \multirow[b]{2}{*}{$\mathrm{t}$} & \multirow[b]{2}{*}{ ddl } & \multirow[b]{2}{*}{ Sig. (bilatéral) } & \multirow{2}{*}{$\begin{array}{l}\text { Différence } \\
\text { moyenne }\end{array}$} & \multirow{2}{*}{$\begin{array}{c}\text { Différence } \\
\text { erreur } \\
\text { standard }\end{array}$} & \multicolumn{2}{|c|}{$\begin{array}{l}\text { Intervalle de confiance de la } \\
\text { différence à } 95 \%\end{array}$} \\
\hline & & & & & & & & & Inférieur & Supérieur \\
\hline \multirow[t]{2}{*}{ Salaire du coach } & $\begin{array}{l}\text { Hypothèse de variances } \\
\text { égales }\end{array}$ & 3,416 & 083 & $-2,604$ & 16 &, 019 & $-1122222,222$ & 430886,969 & $-2035661,792$ & $-208782,653$ \\
\hline & $\begin{array}{l}\text { Hypothèse de variances } \\
\text { inégales }\end{array}$ & & & $-2,604$ & 10,193 &, 026 & $-1122222,222$ & 430886,969 & $-2079841,327$ & $-164603,117$ \\
\hline
\end{tabular}

Tableau 6 - Test T salaire entraîneur-chef - changement

Pour évaluer l'influence du changement d'entraîneur-chef sur la performance d'une équipe, nous avons comparé les deux situations suivantes : 1) la performance de l'entraîneur-chef initial de la dernière année de responsabilité avec celle de la première année de responsabilité de l'entraîneur-chef remplaçant et; 2) la moyenne de la performance de l'entraîneur-chef initial avec la moyenne de la performance de l'entraîneur-chef remplaçant durant leurs années respectives de responsabilité.

Dans le premier cas, les résultats du tableau 7 montrent que la moyenne de la performance au cours de la dernière année des entraîneurs-chefs initiaux 
$(79,65)$ est plus faible que celle des entraîneurschefs remplaçants $(85,08)$. Cependant, le test $\mathrm{T}$ des échantillons indépendants du tableau 8 montre qu'il n'existe pas de différence significative (Sig ,315) entre la performance des dernières années des entraîneurs-chefs initiaux et ceux des premières années des entraîneurs-chefs remplaçants au cours des saisons 2012 à 2017. L'absence de lien entre le changement d'entraîneur et la performance de l'équipe à court terme pourrait s'expliquer par le fait qu'une équipe qui occupe les dernières places dans un classement, même avec un changement d'entraîneur-chef, ne peut dansl'immédiat améliorer son classement de l'année.

\section{Statistiques de groupe}

\begin{tabular}{|c|c|c|c|c|c|}
\hline & Changement de Coach & $N$ & Moyenne & Ecart type & $\begin{array}{l}\text { Moyenne } \\
\text { erreur } \\
\text { standard }\end{array}$ \\
\hline \multirow[t]{2}{*}{ Performance équipe } & Entraineur initial & 26 & 79,65 & 23,921 & 4,691 \\
\hline & Entraineur remplaçant & 26 & 85,08 & 13,007 & 2,551 \\
\hline
\end{tabular}

Tableau 7 - Statistiques descriptives salaire entraîneur-chef - performance

\begin{tabular}{|c|c|c|c|c|c|c|c|c|c|c|}
\hline \multicolumn{11}{|c|}{ Test des échantillons indépendants } \\
\hline & & \multicolumn{2}{|c|}{$\begin{array}{c}\text { Test de Levene sur l'égalité } \\
\text { des variances }\end{array}$} & \multicolumn{7}{|c|}{ Test t pour égalité des moyennes } \\
\hline & & \multirow[b]{2}{*}{$\mathrm{F}$} & \multirow[b]{2}{*}{ Sig. } & \multirow[b]{2}{*}{$\mathrm{t}$} & \multirow[b]{2}{*}{ ddl } & \multirow[b]{2}{*}{ Sig. (bilatéral) } & \multirow{2}{*}{$\begin{array}{c}\text { Différence } \\
\text { moyenne }\end{array}$} & \multirow{2}{*}{$\begin{array}{l}\text { Différence } \\
\text { erreur } \\
\text { standard }\end{array}$} & \multicolumn{2}{|c|}{$\begin{array}{l}\text { Intervalle de confiance de la } \\
\text { différence à } 95 \%\end{array}$} \\
\hline & & & & & & & & & Inférieur & Supérieur \\
\hline \multirow[t]{2}{*}{ Performance équipe } & $\begin{array}{l}\text { Hypothèse de variances } \\
\text { égales }\end{array}$ & 17,803 & .000 & $-1,016$ & 50 & .315 & $-5,423$ & 5,340 & $-16,149$ & 5,303 \\
\hline & $\begin{array}{l}\text { Hypothèse de variances } \\
\text { inégales }\end{array}$ & & & $-1,016$ & 38,595 & .316 & $-5,423$ & 5,340 & $-16,228$ & 5,382 \\
\hline
\end{tabular}

Tableau 8 - Test T salaire entraîneur-chef - performance

Dans le deuxième cas, les résultats du tableau 9 montrent que la performance moyenne de l'entraîneur initial $(73,6)$ est plus faible que celle de son successeur $(87,66)$. Le test $\mathrm{T}$ des échantillons indépendants du Tableau 10 montre qu'il existe une différence significative (Sig ,002) entre la performance moyenne des entraîneurs-chefs initiaux et ceux qui les remplacent au cours des saisons 2012 à 2017. À moyen terme (deux à trois ans) la différence entre la performance des deux groupes d'entraîneurs s'accentue de manière significative.

\section{Statistiques de groupe}

\begin{tabular}{|c|c|c|c|c|c|}
\hline & Changement de Coach & $N$ & Moyenne & Ecart type & $\begin{array}{l}\text { Moyenne } \\
\text { erreur } \\
\text { standard }\end{array}$ \\
\hline \multirow[t]{2}{*}{ Performance équipe } & Entraineur initial & 26 & 73,67 & 18,896 & 3,706 \\
\hline & Entraineur remplaçant & 26 & 87,66 & 11,026 & 2,162 \\
\hline
\end{tabular}

Tableau 9-Statistiques descriptives changement - performance 




Tableau 10 - Test $T$ et changement - performance

\section{Discussion et limites}

À la lecture des résultats des analyses statistiques de notre échantillon, les tendances suivantes ressortent :

1. Le salaire de l'entraîneur-chef d'une équipe de la LNH n'a pas d'influence sur les résultats de l'équipe (performance).

2. À court terme le remplacement d'un entrấneur-chef n'a pas d'incidence sur la performance de l'équipe, mais pourrait avoir un impact à moyen terme.

3. Le lieu d'installation d'une équipe n'a pas d'influence sur la performance de l'équipe.

\section{Les entraîneurs-chefs remplaçants sont mieux} rémunérés que ceux qui ont été congédiés.

La première hypothèse de notre recherche selon laquelle le niveau de salaire des entraineurs-chefs a un impact sur la performance des équipes de bockey (H1) ne peut être vérifiée par nos analyses. La seconde hypothèse qui stipule que le remplacement des entraineurs-chefs a une incidence sur la performance des équipes de bockey (H2) peut être vérifiée selon une certaine condition temporelle (moyen terme, deux à trois ans). Le nouvel entraîneur mieux payé que l'entraîneur congédié ne contribue à la performance de son équipe que quelques années plus tard. Ce qui est logique étant donné la meilleure compréhension de l'équipe par le nouvel entraîneur et l'adaptation des joueurs au nouveau style de jeu.

Comme décrit dans la revue de la littérature, le salaire des entraîneurs-chefs ne semble pas toujours corrélé avec la performance des équipes (Mixon et Wright, 2013). Dans le sport universitaire américain, les résultats divergent quelque peu du sport professionnel puisque certains chercheurs (Colbert et Eckard 2015) semblent établir une corrélation positive entre le salaire du coach et la performance de l'équipe. Quant au remplacement d'un entrâ̂neur-chef, Colbert et Eckard (2013) montrent que cette stratégie est bénéfique à moyen terme. Notre étude quant à elle s'inscrit dans le cas d'une ligue professionnelle $(\mathrm{LNH})$ et les résultats montrent que les déterminants de la performance liés au coach ne semblent pas être similaires pour toutes les ligues de sport. Il y a lieu de faire d'autres études sur d'autres types de sport universitaire et professionnel afin de valider les résultats de la recherche avec un échantillon plus représentatif.

Notre base de données étant constituée de diverses sources d'informations secondaires sur une courte période de cinq ans, nous sommes conscients de certaines limites de notre analyse. De meilleurs résultats pourraient être obtenus en disposant d'informations officielles sur une plus longue durée. De futures recherches réalisées en partenariat avec les ligues de sport universitaire ou professionnel permettraient une meilleure accessibilité aux données et des analyses plus pertinentes et significatives.

\section{Conclusion}

Les résultats de notre présente étude, bien qu'exploratoires, contribuent à documenter la littérature scientifique sur les déterminants de la performance des équipes professionnelles, particulièrement dans le cas de la LNH. Bien que les déterminants de la performance d'une équipe de sport professionnel soient nombreux et multifactoriels tels que la qualité des joueurs, la qualité du management, la qualité de l'entraîneur, les motivations, le soutien par les partisans et d'autres facteurs micro, méso et macroenvironnementaux, notre contribution porte sur le fait que les salaires des entraineurs-chefs ne semblent pas affecter significativement la performance des équipes de la LNH. Néanmoins, nous 
constatons que la plupart des changements d'entraîneurs-chefs effectués durant ou après la saison ont un impact positif à moyen terme sur la performance des équipes de la LNH. Comme dans toute organisation, les facteurs de motivation se trouvent dans des variables contextuelles. Les changements d'entraîneurs-chefs s'inscrivent dans cette optique et apportent une nouvelle dynamique qui accentue la motivation dans l'organisation, ce qui ultimement améliore la performance des équipes.
Dans les équipes professionnelles, les entraîneurschefs sont la plupart du temps choisis par les directeurs généraux, communément appelés General managers, qui prennent les principales décisions sportives comme le congédiement d'un entraîneur-chef, par exemple. Il serait donc très intéressant de mettre l'accent sur les directeurs généraux dans une prochaine étude afin de voir leur contribution à la performance d'équipe de sport professionnel.

\section{RÉFÉRENCES}

Andersen, J. A. (2011). A new sports manager does not make a better team. International Journal of Sports Science \& Coaching, 6(1), 167-178.

Balduck, A-L., Buelens, M. et Philippaerts, R. (2010). Short-term effects of midseason coach turnover on team performance in soccer. Research Quarterly for Exercise and Sport, 81(3), 379-383.

Bormann, K. C., Schulte-Coerne, P., Diebig, M. et Rowold, J. (2016). Athlete characteristics and team comptetive performance as moderators for the relationship between coach transformational leadership and athlete performance. Journal of Sport \& Exercise Psychology, 38, 268-281.

Colbert, G. J. et Woodrow Eckard, E. (2015). Do college get what they pay for? Evidence on football coach pay and team performance. Journal of Sports Economics, 16(4), 335-352.

Darvin, L., Pegoraro, A. et Berri, D. (2017). «Are men better leaders? An investigation of head coaches'gender and individual players' performance in amateur and professional women's basketball. Sex Roles, 1-12.

Frick, B. et Simmons, R. (2008). The impact of managerial quality on organizational performance: Evidence from german soccer. Managerial and Decision Economics, 29, 593-600.

Grant, R. R., Leadley, J. C. et Zygmont, Z. X. (2013). Just win baby? Determinants of NCAA football subdivision coaching compensation. International Journal of Sport Finance, 8, 61-74.

Idson, T. L. et Kahane, L. H. (2000). Team effects on compensation: an application to salary determination in the National Hockey League. Economic Inquiry, 38(2), 345-357.

Knoppers, A., Meyer, B. B., Ewing, M. et Forrest, L. (1991). Opportunity and work behavior in college coaching. Journal of Sport and Social Issues, 15(1), 1-20.

Koening, R. H. (2003). An econometric evaluation of the effect of firing a coach on team performance. Applied Economics, 35, 555-564.

Lago-Ballesteros, J. et Lago-Penos, C. (2010). Performance in team sports : identifying the keys to success in soccer. Journal of Human Kinetics, 25, 85-91.

Llorca, M. et Teste, T. (2016). Dépenses salariales et performance dans l'industrie du football. Revue Française d'Économie, 31(2), $125-145$.

Martinez, J. A. et Caudill, S. B. (2013). Does midseason change of coach improve team performance? Evidence from the NBA. Journal of Sport Management, 27, 108-113.

Mixon, P., Byrd, W. J. et Wright, A. (2013). Does pay lead to performance? Using NCAA head football coaches as a surrogate for CEOS. Journal of Business and Behavioral Sciences, 25(2), 25-37.

Mondello, M. et Maxcy, J. (2009). The impact of salary dispersion and performance bonuses in NFL organizations. Management Decision, 47(1), 110-123.

Slattery, K. M., Wallace, L. K., Bentley, D. J. et Coutts, A. J. (2012). Effect of training load on simulated team sport match performance. Applied Physiology, Nutrition, and Metabolism, 37(2), 315-322. 
Solomon, G. B. (2002). Source of expectancy information among assistant coaches: the influences of performance and psychological cues. Journal of Sport Behavior, 25(3), 279-286.

Vela, S. A., Oades, L. G. et Crowe, T. P. (2013). The relationship between coach leadership, the coach - athlete relationship, team success, and the positive developmental experiences of adolescent soccer players. Physical Education and Sport Pedagogy, 18(5), 549-561.

White, P., Persad, S. et Gee, C. J. (2007). The effect of mid-season coach turnover on team performance: the case of the National Hockey League (1983-2003). International Journal of Sports Science \& Coaching, 2(2), 143-152. 